\title{
Fernando Ginés: la despedida a un colega entrañable
}

\author{
Dres. Tomás Dieste', Ricardo Lluberas², Jorge Mayol', \\ Andrés Tuzman², Pablo Vázquez ${ }^{1}$
}

El Consejo Editorial de la Revista Uruguaya de Cardiología nos ha encomendado realizar un recordatorio de la figura del Prof. Agdo. Dr. Fernando Ginés.

Es habitual que un obituario sea realizado por una sola persona. En este caso, como la figura de Fernando fue tan querida, se dio la situación de que varios de sus compañeros de trayectoria quisieran participar. Y así fue. Este es un recuerdo de Fernando realizado colectivamente entre quienes aprendieron con él y que lo recordarán como el buen médico y persona que fue.

Fernando (el Pupi o el Petiso) Ginés fue un personaje entrañable. Irradiaba simpatía y buena onda, por lo que hacía virtualmente imposible que no nos despertara cariño.

Pero su figura no era solamente eso. Se agregaban una vasta experiencia y sólida formación.

Desarrolló una larga carrera en Ciencias Básicas, donde culminó siendo profesor agregado de Fisiología. Muchas generaciones de médicos recibie-

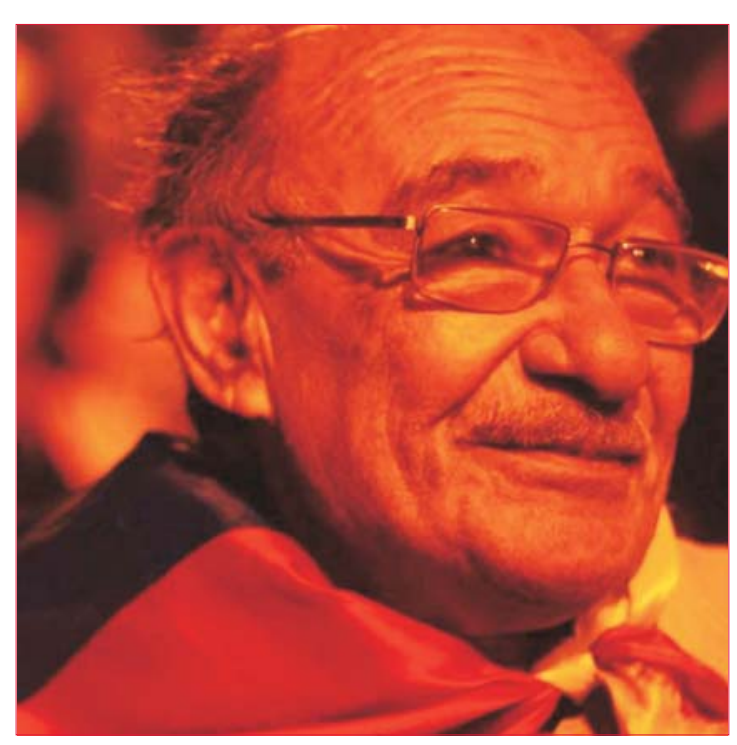

Prof. Agdo. Dr. Fernando Ginés ron sus enseñanzas en fisiología cardiovascular.

Probablemente muchos de nosotros, cardiólogos, despertamos el interés por la especialidad con esas clases.

Uno de los temas que estudió con profundidad, junto a su equipo de la Cátedra de Fisiología, fue sobre el comportamiento del ventrículo derecho en experimentación animal. Publicaron numerosos trabajos en congresos y revistas, tanto nacionales como internacionales. Uno de ellos fue presentado en el Congreso Uruguayo de Cardiología de 1988 y llevaba como nombre El ventrículo derecho: ¿̇hermano menor en el sistema cardiocirculatorio? Un nombre provocador y que siempre recordamos, especialmente cuando nos toca asistir en la actualidad a pacientes en shock cardiogénico por falla de aquel ventrículo, en el que generalmente menos pensamos los cardiólogos.

Fue además, en su práctica clínica, un cardiólogo de enorme experiencia.

Con Ginés se dio el aprendizaje inicial de muchos cardiólogos intervencionistas que trabajan en Uruguay. Buena parte de nuestro entrenamiento en cateterismos braquiales, por la vieja técnica de Sones, lo realizamos con Fernando a nuestro lado.

Así también ocurrió con los cateterismos derechos. Los cardiólogos intervencionistas más veteranos pertenecemos a una época en que esta técnica era habitual en la sala de hemodinamia. Era la rutina que un paciente con patología valvular recibiera un estudio de su eventual repercusión derecha. Y ni que hablar de los pacientes con cardiopatía congénita.

1. Centro Cardiológico Americano, Sanatorio Americano. Montevideo, Uruguay.

2. Instituto de Cardiología Intervencionista Casa de Galicia y Cardiocentro, Asociación Española. Montevideo, Uruguay. 
En ambos casos, hacíamos los estudios frecuentemente con Fernando, que no perdía nunca esa actitud de buen compañero, con el que era un gusto trabajar. Siempre tenía gran disposición docente y por supuesto mucha información que estaba dispuesto a compartir.

Fue también un cardiólogo orientado a la cardiología pediátrica. La practicó desde el punto de vista clínico durante muchos años de su vida, y en la sala de hemodinamia fue uno de los impulsores de esta disciplina. Podrán imaginar lo que aprendíamos los más jóvenes viendo y ayudando a colegas como él y Carlos Peluffo, los miércoles en la sala del INCOR, cuando se practicaban cateterismos en patologías congénitas.

Su pasión por la cardiología pediátrica lo llevó a participar durante muchos años en la docencia de esta disciplina, y a colaborar en forma muy importante en la concreción del curso de posgrado de esta especialidad.

Como este es un documento que quiere recordar al Ginés que todos vivimos, cada uno de los autores aportó algunos recuerdos o anécdotas que "lo pintan" tal cual era.

Fernando nació en Asunción, Paraguay. Si bien se había convertido en un uruguayo más, mantenía un cierto tonito que lo distinguía.

Lo otro que lo mostraba como paraguayo era que tomaba tereré. Varios de nosotros aprendimos con él a beber esa infusión helada, muy alejada de nuestras costumbres gauchescas. Y luego llevábamos la bebida a la Unidad Cardiológica donde, en general, era rechazada por nuestros demás compañeros...

Tomás Dieste. Llegué al INCOR en 1983. Los que dirigían el servicio eran Fausto Buitrón y Fernando Ginés. Fausto más dedicado a los pacientes adultos y Fernando a los congénitos.

Con Ginés realicé mi primera valvuloplastia pulmonar con técnica de doble balón y recibimos todo el empuje y la confianza para dedicarnos al diagnóstico e intervencionismo cardiovascular.

Supo dar un paso al costado y nos cedió el espacio para que creciéramos, ayudando a Fausto, en el desarrollo de la angioplastia coronaria.

Otra demostración de generosidad fue cuando Carlos Peluffo regresó de su exilio en Suecia, y Fernando le abrió las puertas de la sala como si nunca se hubiera ido.

Enriquecía las discusiones clínicas tanto de congénitos como de adultos.

A veces desaparecía y la barra se preguntaba ¿dónde está el Petiso?

Era fácil ubicarlo. En su querido Departamento de Fisiología, donde fue reintegrado a la vuelta de la democracia.

Ricardo Lluberas. En nuestra memoria está un caso clínico que ocurrió cercano a los años 1983-1984. Habíamos recibido un paciente joven, de unos 40 años, que había sufrido un infarto inferior en el interior del país. Fue enviado en avión a Montevideo luego de la etapa aguda.

Cuando Fernando fue a estudiar la coronaria derecha, hizo la clásica maniobra de rotar el catéter de Sones en sentido horario para entrar en la arteria, y ocurrió lo que a veces sucedía con esta maniobra: la punta del catéter terminó en el tercio medio de la arteria. Ahí comprobamos que la arteria era aneurismática a nivel proximal y estaba ocluida por un notorio trombo en el tercio medio.

Fernando miró a su ayudante, quien inmediatamente adivinó su intención. Luego de que el ayudante le dijera "dale", Fernando atravesó el trombo con la punta del catéter... iY la arteria quedó permeable!

Habíamos hecho una reperfusión mecánica en el infarto. No lo publicamos y quedó en el anecdotario. ¡Hubiera sido la documentación temprana de un procedimiento que años después se transformó en rutina en el manejo del infarto!

Jorge Mayol. Recuerdo que Fernando se había formado en cardiología y posteriormente en hemodinamia, en el Instituto Dante Pazzanese de San Pablo, y era uno de los dos referentes del equipo de Hemodinamia del INCOR.

A fines de los años 80 fue muy importante una visita al servicio por parte del Prof. Dr. Eduardo de Souza, director del Dante Pazzanese y referente mundial en el intervencionismo coronario. De Souza había venido a enseñar sobre angioplastia coronaria. Era una persona muy formal y generaba mucho respeto en su entorno, pero fue muy llamativo el cambio de su postura cuando se encontró con Fernando. Surgieron las anécdotas sobre la pasantía de Ginés por "el Dante” y llegó a recordar el apodo con el que por allí se lo conocía: "Vadinho"*.

* Personaje de la novela de Jorge Amado, Doña Flor y sus dos maridos, caracterizado por su simpatía y alegría de vivir. 
Esa fue una clara muestra de la simpatía que Fernando lograba irradiar en cada uno de los lugares donde le tocaba estar.

Pablo Vázquez. Recuerdo mi primer día en el INCOR, por agosto de 1995. Era para mí todo un desafío. Recién recibido, cero experiencia, y con todas las dudas que significaba este reto. Fue ahí mi primer contacto con Fernando. Yo, sentado, observando todo desde la sala de control, con el Negrito Tuzman (que ya hacía unos meses que había llegado al servicio) y el Gallego Manolo, tratando de explicarme el funcionamiento de la sala que para mí, un canario de Durazno, era la NASA... y en ese momento irrumpe Fernando, despilfarrando alegría, haciendo chistes, me mira y me dice: "Gurí, vos sos el hijo del Lucho Vázquez de Durazno"? Yo le contesto "sí"... me da un abrazo y me dice: "Tu viejo fue profesor mío, de clínica pediátrica, y me enseñó mucho de pediatría en el Pedro Visca, así que yo te voy a enseñar a vos a hacer cateterismos".

De ahí en más, Fernando se transformó en mi maestro de Sones.

Primero aprender a disecar el paquete humeral, luego cateterismo derecho y ya cuando estábamos más adiestrados, pasamos a realizar el cateterismo izquierdo.

Siempre con buen humor, con risas, sin tensión, con mucha paciencia y con enorme docencia.

Muchas de sus enseñanzas me quedaron para toda la vida.

Solo quiero comentar una, que la practico mucho y a veces, cuando no lo hago, la realidad del trabajo se encarga de recordármelo. Él siempre me decía: “cuando la rutina te lleve a tener que hacer cinco o seis cateterismos seguidos en un día, no te olvides de pensar, aunque más no sea un minuto, cuando te estés lavando para entrar, en qué se me puede complicar este paciente", y agregaba: "porque no es lo mismo que la complicación te sorprenda a que hayas pensado en ella".

Hoy nos toca despedirte Pupi. Saludamos a ese Petiso bueno y querible que fuiste, con quien tanto aprendimos cuando veíamos a la cardiología como algo enorme a conocer. Así te recordaremos.

Tomás Dieste, https://orcid.org/0000-0003-3825-1641

Ricardo Lluberas, https://orcid.org/0000-0003-2512-9356

Jorge Mayol, https://orcid.org/0000-0002-7739-287X

Andrés Tuzman, https://orcid.org/0000-0002-7391-8213

Pablo Vázquez, https://orcid.org/0000-0001-7933-3964 